\title{
Thermoelectric Properties of Composite Nanoparticle Array with Protoporphyrin Molecule Junctions
}

\author{
I-Chih $\mathrm{Ni}^{1}$, and Shien-Der Tzeng ${ }^{1}$ \\ ${ }^{1}$ Department of Physics, National Dong Hwa University, 97401 Shoufeng, Hualien, Taiwan, R.O.C.
}

\begin{abstract}
Composite nano-structures have many interesting properties and potential applications. For a moleculenanoparticle (NP) composite array, its properties may strongly depend on the molecules on/between NPs. For instance, the resistance of NP composite film sensitively change with the length of surface molecules, ${ }^{[1,2]}$ and could be applied to vapor sensors. ${ }^{[3]}$ In this report, we show a homemade system to measure thermoelectric properties of protoporphyrin molecule junctions in molecule-NP composite array. Many kinds of metal-protoporphyrin molecules (Heme, Hematin, Zinc-protoporphyrin, Cobaltic-protoporphyrin, Protoporphyrin IX) and dl-cysteine/cysteamine molecules were studied. We found metal ion in protoporphyrin molecule is matter for the Seebeck coefficient of molecule-NP composite array.
\end{abstract}

\section{Introduction}

Recent years, thermoelectric properties of molecule junctions were reported in theories ${ }^{[4-6]}$ and in experiments ${ }^{[7-9]}$. For instance, $\mathrm{Yu}$-Shen Liu et al. presented the thermoelectricity in molecular junctions formed by a single molecule contact through firstprinciple calculation. ${ }^{[4]}$ The Seebeck coefficient of molecule-metal junction is related to the slope of the transmission function near Fermi level, and could be described by eq. (1).

$$
\mathrm{S}=-\left.\frac{\pi^{2} K_{B}{ }^{2} T}{3 e} \frac{\partial \ln \tau(E)}{\partial E}\right|_{E=E_{F}} .
$$

Here $\tau(E)$ is transmission function of electron with energy $E$ passing through molecule junction between electrodes. The Seebeck coefficient would be equal to zero when peak position of LUMO or HOMO aligns with the Fermi level $\left(E_{F}\right)$. In addition, Seebeck coefficients are related to the structure and functional group of molecules. ${ }^{[9]}$ For instance, amine-terminated molecule junctions may have positive Seebeck coefficient because of HOMO-conducting, while pyridine-terminated molecule junctions have negative Seebeck coefficient because of LUMO-conducting. B. Wang et al. reported calculated thermoelectric property of different length of alkane molecules between Au electrodes. ${ }^{[6]}$ They found the thermoelectric voltages are positive when the number of carbon is odd, while the voltages is negative when the number of carbon is even.

Recent years, scanning probe microscopy had been used to measure thermoelectric properties of molecule junctions. ${ }^{[4-5,7-8]}$ In a scanning tunneling microscope (STM), Au tip is kept at room temperature with a large

\footnotetext{
a Corresponding author: author@e-mail.org
}

thermal reservoir as a cool side; Au substrate is heated to a controlled temperature, then the temperature difference $(\mathrm{d} T)$ is formed between substrate and tip. The $\mathrm{Au}$ substrate surface has some thiol-terminated molecules, then $\mathrm{Au}$ STM tip slowly moves to approach these molecules by monitoring tunneling current between tip and substrate. After tip traps molecules, tip slowly withdraw to a sufficiently large distance. Meanwhile, thermoelectric voltage is measured. Although, STM measurement could be capable of measuring the Seebeck coefficient of single molecule, its operation is relatively difficult and STM is expensive.

In this report, we use gold nanoparticle (AuNP) array to form abundant molecule junctions, and measure their thermoelectric properties. Our measurement method is simple, inexpensive, convenient, stable, and can provide rich molecule-NP nano-composite structures for future applications.

\section{Basic Principles}

Thermoelectric voltage of molecule junctions could come from differential Fermi levels of left and right molecule-electrode junctions. ${ }^{[4-6,10-12]}$ In our experiment, electrodes are AuNPs, and metal-protoporphyrin molecules are modified on AuNP surfaces. When thermoelectric voltage is created, the current of molecule junction could be described with Landauer function:

$$
\mathrm{I}=\frac{2 q}{h} \int \overline{T(E)}\left(f_{1}-f_{2}\right) d E
$$

Here $\overline{T(E)}$ is the transmission function, and $f_{1}$ and $f_{2}$ are equilibrium Fermi-Dirac distributions for heating and 
cooling contacts. Fermi level $f_{1}-f_{2}$ could be described by

$$
\begin{gathered}
f_{1}-f_{2} \approx \frac{\partial f}{\partial \mu} \Delta \mu+\frac{\partial f}{\partial T} \Delta T \\
f(\mu, T)=\frac{1}{e^{(E-\mu) / K_{B} T}+1}
\end{gathered}
$$

Here $\Delta T=T_{1}-T_{2}$ is the temperature difference, and $\Delta \mu$ is the Fermi level difference between contacts. The current of molecule junction could be described by

$$
\begin{gathered}
I=\frac{2 q}{h} \int \overline{T(E)}\left(\frac{\partial f}{\partial \mu} \Delta \mu+\frac{\partial f}{\partial T} \Delta T\right) d E \\
\frac{\partial f}{\partial \mu}=e^{\frac{E-\mu}{K_{B} T}} \cdot \frac{1}{K_{B} T}\left(e^{\frac{E-\mu}{K_{B} T}}+1\right)^{-2} \\
\frac{\partial f}{\partial T}=e^{\frac{E-\mu}{K_{B} T}} \cdot \frac{(E-\mu)}{K_{B} T^{2}}\left(e^{\frac{E-\mu}{K_{B} T}}+1\right)^{-2}=\frac{E-\mu}{T}\left(\frac{\partial f}{\partial E}\right)
\end{gathered}
$$

For linear response regime, heating and cooling rates of contacts are equal, the current of molecule junction could be described by

$$
\mathrm{I} \approx<\mathrm{G}>\Delta \mathrm{V}+<\mathrm{SG}>\Delta T
$$

Here $\mathrm{S}$ is Seebeck coefficient, $\mathrm{G}$ is conductance, and

$$
<\mathrm{G}>\Delta \mathrm{V}=\frac{2 q}{h} \int \overline{T(E)}\left(\frac{\partial f}{\partial \mu} \Delta \mu\right) d E
$$

According to $\Delta \mu=-q \times \Delta V, \Delta V=V_{1}-V_{2}$,

$$
\begin{gathered}
<\mathrm{G}>\Delta \mathrm{V}=\frac{2 q^{2}}{h} \int \overline{T(E)}\left(-\frac{\partial f}{\partial E} d E\right) \Delta V \\
<\mathrm{G}>=\frac{2 q^{2}}{h} \int \overline{T(E)}\left(-\frac{\partial f}{\partial E} d E\right)
\end{gathered}
$$

Another part,

$$
\begin{gathered}
<\mathrm{SG}>\Delta T=\frac{2 q}{h} \int \overline{T(E)}\left(\frac{\partial f}{\partial T} \Delta T\right) d E \\
=-\frac{2 q}{h T} \int \overline{T(E)}(E-\mu)\left(-\frac{\partial f}{\partial E}\right) \cdot d E(\Delta T) \\
<\mathrm{SG}>=-\frac{2 q}{h T} \int \overline{T(E)}(E-\mu)\left(-\frac{\partial f}{\partial E}\right) \cdot d E
\end{gathered}
$$

Then, Seebeck coefficient could be described by

$$
\begin{aligned}
\mathrm{S}=\frac{<\mathrm{SG}>}{<\mathrm{G}>} & =\frac{-\frac{2 q}{h T} \int \overline{T(E)}(E-\mu)\left(-\frac{\partial f}{\partial E}\right) \cdot d E}{\frac{2 q^{2}}{h} \int \overline{T(E)}\left(-\frac{\partial f}{\partial E} d E\right)} \\
& =\frac{K_{B}}{-q} \frac{\int \overline{T(E)} \frac{\left(E-E_{F}\right)}{K_{B} T}\left(-\frac{\partial f}{\partial E}\right) \cdot d E}{\int \overline{T(E)}\left(-\frac{\partial f}{\partial E} d E\right)}
\end{aligned}
$$

Because

$$
\frac{\partial f}{\partial T}=\frac{E-\mu}{T}\left(\frac{\partial f}{\partial E}\right), \frac{\partial f}{\partial T}=-\left(\frac{\partial f}{\partial E}\right) \delta(E-\mu)
$$

Then

$$
\begin{aligned}
\frac{\Delta \mu}{\Delta T} & =\left.\frac{1}{T(E)} \int \overline{T(E)} \frac{(E-\mu)}{T}\left(\frac{\partial f}{\partial E}\right) d E\right|_{E=u} \\
& =\left.\frac{\pi^{2} K_{B}{ }^{2} T}{3} \frac{1}{\overline{T(E)}} \frac{\partial \overline{T(E)}}{\partial E}\right|_{E=\mu}
\end{aligned}
$$

Finally, Seebeck coefficient could be described by

$$
\mathrm{S}=-\frac{\Delta V}{\Delta T}=-\left.\frac{\pi^{2} K_{B}{ }^{2} T}{3 e} \frac{1}{\overline{T(E)}} \frac{\partial \overline{T(E)}}{\partial E}\right|_{E=\mu}
$$

\section{Experiment method and measurement system}

\subsection{Metal-protoporphyrin-AuNP film}

AuNP surfaces are modified with metalprotoporphyrin molecules (such as Heme, Hematin, Zincprotoporphyrin, Protoporphyrin IX, and Cobalticprotoporphyrin) or dl-cysteine/cysteamine molecules, then deposited on glass substrate surface with symmetrical electrodes by a centrifuge deposition method. ${ }^{[1]}$ The symmetrical electrodes were $20 \mathrm{~nm} / 50 \mathrm{~nm}$ $\mathrm{Cr} / \mathrm{Au}$ deposited on glass substrate (Corning). Highpurity $\mathrm{Cu}$ wires were adhered respectively on two pairs of electrodes by silver filled electrically conductive adhesives.

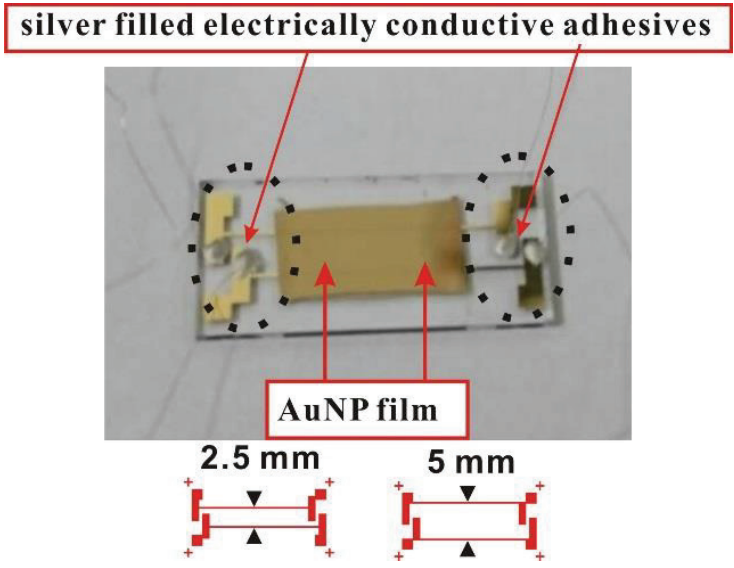

Figure 1. Sketch images of electrodes, and a picture of molecule-AuNP film on a $2.5 \mathrm{~mm}$-gap electrodes.

\subsection{Measurement system}

We built up a system for thermoelectric voltage measurements with high sensitivity $(0.1 \mu \mathrm{V})$ and low thermal noise for high resistance samples (to $300 \mathrm{k} \Omega$ ). The design of homemade measurement system is shown in figure 2. Measurement instruments and data acquisition are connected and controlled by LabVIEW program. The vacuum pressure of system chamber is about $10^{-5}$ torr. Sample is placed on a metal bar connected between heater and cooler stages. In experiment, the initial temperatures of hot and cold stage are kept at about $150{ }^{\circ} \mathrm{C}$ and $4{ }^{\circ} \mathrm{C}$ respectively, and then slowly return to room temperature through shutdown the heating and cooling power simultaneously. Thermoelectric voltage of molecule-AuNP film is measured by HP 34420 A microvoltage meter when temperatures slowly return to room temperature. Thermal radiation from heat source is shielded by aluminium foils, as shown in figure 3 . Voltage of thermocouples ( $\mathrm{T}_{\text {cool }}$ and $\mathrm{T}_{\text {hot }}$ ) on both sides of the chip are measured by keithley 2001 and Agilent 34410 A voltage meter. All valves, pumps, gauges, mass 
flows, heater, and cooler are controlled or measured through digital $\mathrm{I} / \mathrm{O}$ and analog $\mathrm{I} / \mathrm{O}$ by a data acquisition board (NI Elvis II).

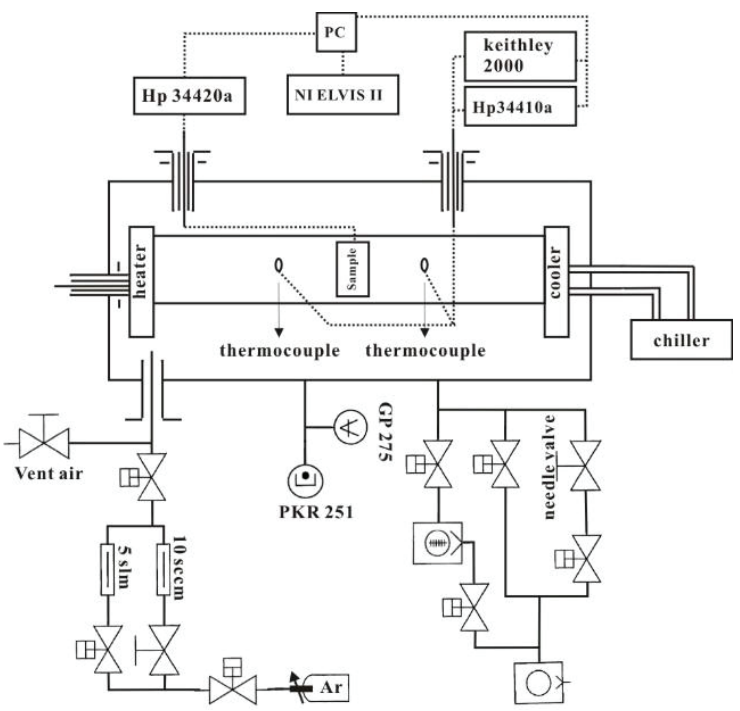

Figure 2. The design of homemade system for molecule thermoelectric measurements.

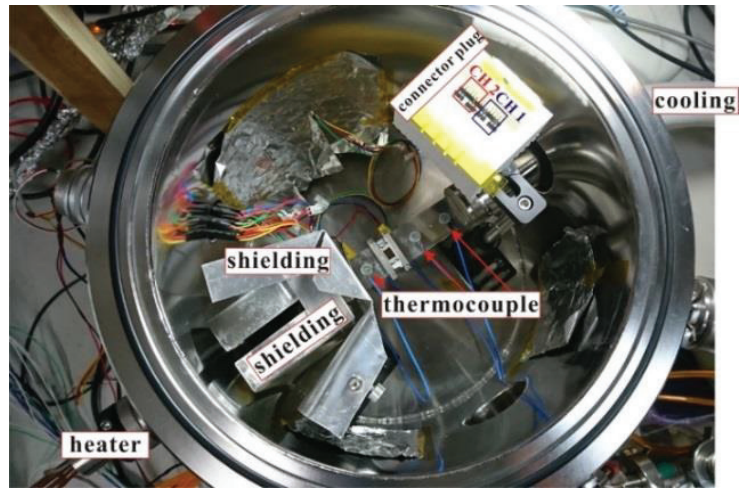

Figure 3. Picture of the measurement system.

\section{Result and Discussion}

Six kinds of molecule-AuNP composite films are studied. Heme, Hematin, Zinc-protoporphyrin, Cobalticprotoporphyrin or Protoporphyrin IX molecules are linked on $\mathrm{NH}_{2}$ function groups of dlcysteine $_{0.75} /$ cysteamine $_{0.25}$-AuNPs by ENC/NHS crosslinking reaction. Figure 4 (a)(c) shows thermoelectric voltage-temperature curves of two HemeAuNP films on symmetrical electrode of $5 \mathrm{~mm}$ and 2.5 $\mathrm{mm}$ gap. Three voltage curves are corresponding to original sample direction $(\mathrm{dV})$, sample rotated with 180 degree $\left(d V_{R}\right)$, and sample lift up from heater bar $\left(d V_{L}\right)$. The curve of $d V-d_{\text {film }}$ is averaged from repeated measurements for 2 3 times. $\mathrm{We}$ found the thermoelectric voltage of $\mathrm{dV}_{\mathrm{L}}$ is near to zero (because $\left.\mathrm{dT}_{\text {film }} \sim 0\right)$. The inverse symmetrical behavior of $\mathrm{dV}$ and $\mathrm{dV}_{\mathrm{R}}$ curves were due to opposite temperture gradient. This displays the acquired voltage are correct thermoelectric voltage from Heme-AuNPs film. Figure 4 (b)(d) show thermoelectric voltage of the AuNP films by eq. (18).

$$
\mathrm{dV}_{\text {film }}=\left(\mathrm{dV}-\mathrm{dV} \mathrm{V}_{\mathrm{R}}\right) / 2
$$

We found the relationship between $\mathrm{dV}_{\text {film }}$ and $\mathrm{dT}_{\text {film }}$ is nearly linear, and the slopes for different gap of symmetrical electrodes are similar. We also calculate the Seebeck coefficient (S) of Heme-AuNP films through eq. (19), and get $\mathrm{S}$ are about 2.5 and $2.4 \mu \mathrm{V} / \mathrm{K}$ for $5 \mathrm{~mm}$ and $2.5 \mathrm{~mm}$ gap electrodes, respectively.

$$
\mathrm{S}=-\frac{\mathrm{dV}_{\text {film }}}{\mathrm{dT}_{\text {film }}}=- \text { slope }
$$
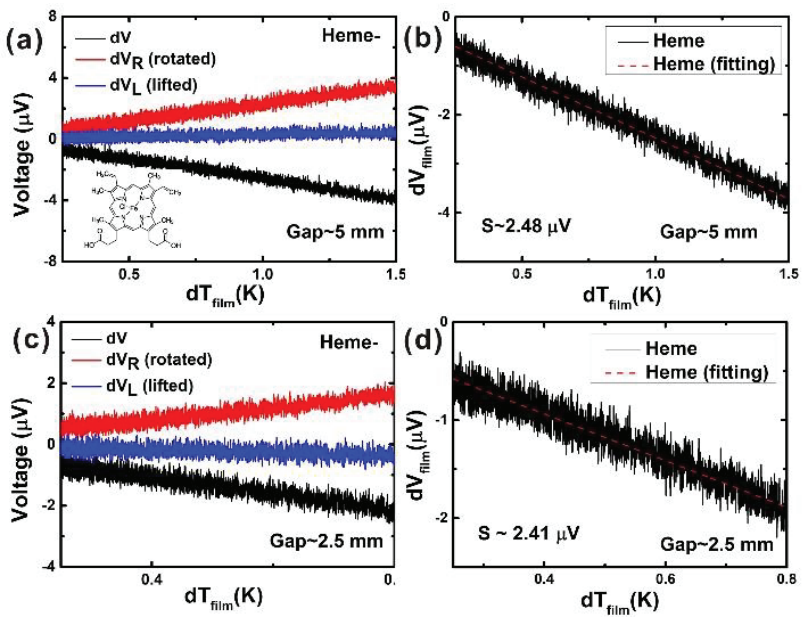

Figure 4. Thermoelectric voltage of Heme-AuNPs films for (a) $5 \mathrm{~mm}$ and (b) $2.5 \mathrm{~mm}$ gap electrodes. (b)(d) $T$ he $\mathrm{dV}_{\text {film- }} \mathrm{dT}_{\text {film }}$ curve. Inset sketch is molecule structure of Heme.

We also found Seebeck coefficients of cysteine ${ }_{0.75} /$ cysteamine $_{0.25}$-AuNP films are negative $(\approx-2.2 \mu \mathrm{V} / \mathrm{K})$. But after linking protoporphyrin molecules on surfaces, its Seebeck coefficient is changed to positive. Table 1 shows Seebeck coefficients of some molecule-AuNPs films (each $\mathrm{S}$ was averaged from $4 \sim 6$ sample). We found Proto-porphrin (no metal ion)-AuNPs films have a Seebeck coefficient $(\mathrm{S} \approx 1.0 \mu \mathrm{V} / \mathrm{K})$ smaller than other metal-Protoporphrin-AuNPs films, and the Seebeck coefficient would increase with electronic configuration of the ceter-matal elements in $3 \mathrm{~d}$ orbital. The Zinc $\left(3 \mathrm{~d}_{10} 4 \mathrm{~s}_{2}\right)$-protoporphyrin samples have largest Seebeck coefficient $(\mathrm{S} \approx 9.6 \mu \mathrm{V} / \mathrm{K})$ than Cobaltic $\left(3 d_{7} 4 s_{2}\right)-$, Heme $\left(3 d_{6} 4 s_{2}\right)-$, and $\operatorname{Hematin}\left(3 d_{6} 4 s_{2}\right)-$ protoporphyrin samples. This may due to its filled $3 \mathrm{~d}$ orbitals. Besides, Heme-AuNP and Hematin-AuNP films have similar Seebeck coefficient. These results show center-metal ion has important influence to the Seebeck coefficient of protoporphyrin molecules.

\section{Conclusion}

We use novel nano-assembly method to fabricate composite molecule-AuNP films and build up a measurement system to study their thermoelectric properties. The composite molecule-AuNP arrays have 
lots of molecule junctions in the films. We got the Seebeck coefficients of metal-protoporphyrin-AuNP films and cysteine/cysteamine-AuNPs films, and found the Seebeck coefficients of metal-protoporphyrin-AuNPs films were positive, but was negative for cysteine/cysteamine-AuNP film. We also found HemeAuNP and Hematin-AuNP films have similar Seebeck coefficient. This may due to the same ceter-metal ion $(\mathrm{Fe})$. Zinc-protoporphyrin have largest Seebeck coefficient than others that may due to its filled $3 \mathrm{~d}$ orbitals.

Table 1. Seebeck coefficient of molecule-AuNP films.

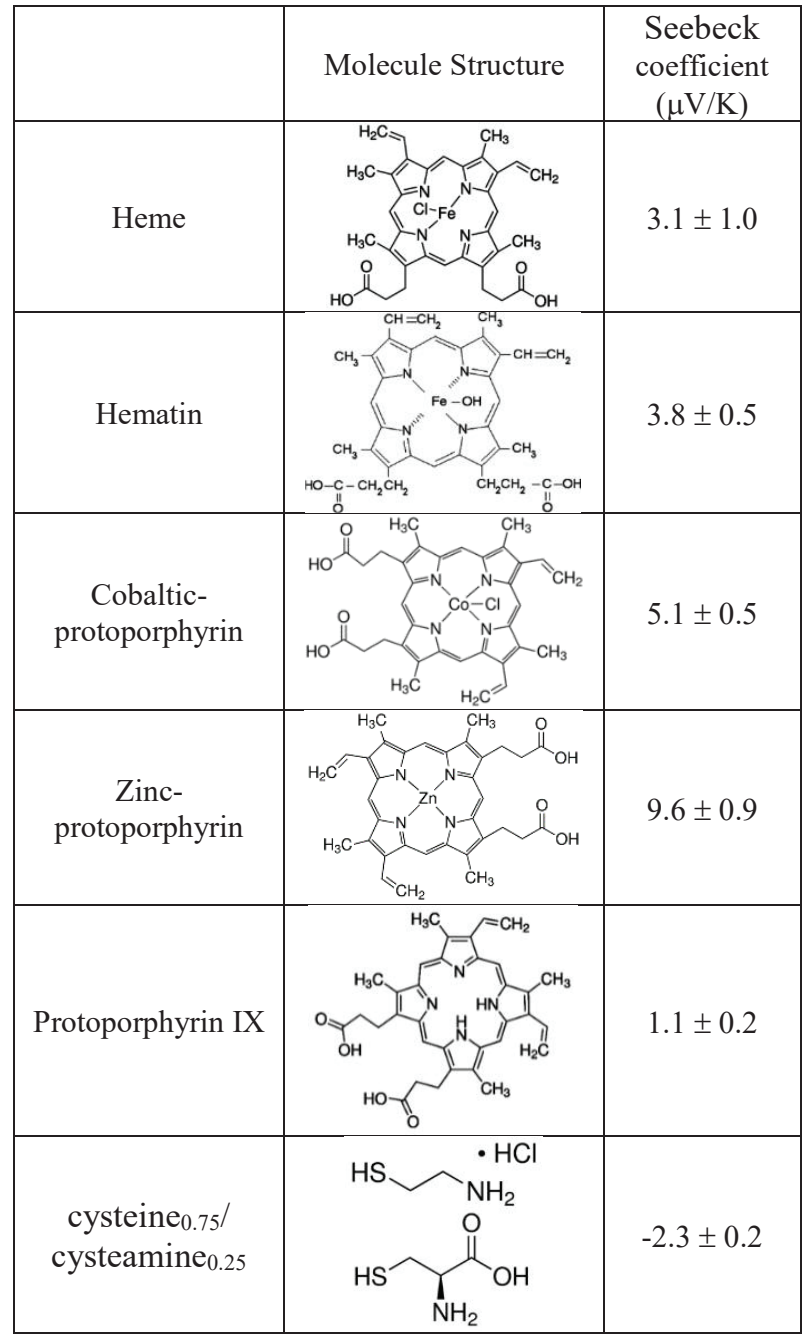

\section{References}

1. C.-W. Jiang, I-C. Ni, S.-D. Tzeng, C.-S. Wu and W. Kuo, Nanoscale 6, 5887-5893 (2014).

2. Amir Z.-K., P.-E. Trudeau, Y. Suganuma, A.-A. Dhirani, and B. Statt, Phys. Rev. Lett. 96, 156403 (2006).

3. Y. Joseph, A. Peić, X. Chen, J. Michl, T. Vossmeyer, and A. Yasuda, J. Phys. Chem. C 111, 12855-12859 (2007).

4. Y.-S. Liu and Y.-C. Chen, Phys. Rev. B 79, 193101 (2009).
5. Y.-S. Liu, Y.-R. Chen and Y.-C. Chen, ACS Nano 3, 3497-2504 (2009).

6. Wang B, Xing YX, Wei YD, Wan LH, Wang J, Carbon 43, 2786-2791 (2005).

7. J. R. Widawsky, W. Chen, H. Vázquez, T. Kim, R. Breslow, M. S. Hybertsen, and L. Venkataraman, Nano Lett. 13, 2889-2894 (2013).

8. P. Reddy, S.-Y. Jang, R. A. Segalman, and A. Majumdar, Science 315, 1568-1571 (2007).

9. J. R. Widawsky, P. Darancet, J. B. Neaton, and L. Venkataraman, Nano Lett. 12, 354-358 (2012).

10. J. P. Bergfield, M. A. Solis, and C. A. Stafford, ACS Nano 4, 5314-5320 (2010)

11. Y. Dubi and M. D. Ventra, Nano Lett. 9, 97-101 (2008).

12. M. Tsutsui and M. Taniguchi, Sensors 12, 7259-7298 (2012). 